\title{
Possible Relationship between Livid Telangiectasis on the Colon Mucosa and Abdominal Kampo Diagnosis
}

\author{
Naoki Mantani, ${ }^{1,3}$ Toshiaki Kogure, ${ }^{1}$ Kin'ichi Fujita, ${ }^{2}$ \\ Jun'ichi Tamura, ${ }^{2}$ Nobuyasu Sekiya, ${ }^{3}$ Yutaka Shimada, ${ }^{3}$ \\ and Katsutoshi Terasawa ${ }^{3}$
}

\begin{abstract}
Backgrounds \& Aims : In daily practice, we have sometimes encountered patients with both Oketsu syndrome and livid telangiectasis on the colon mucosa detected by colonoscopy. The object of this study was to examine the relationship between livid telangiectasis on the colon mucosa and abdominal Kampo diagnosis. Methods : We enrolled consecutive 30 patients with various diseases who underwent total colonoscopy in our department. Clinical characteristics including age, sex, colon adenoma, hemorrhoids and livid telangiectasis on the colon mucosa were examined in each patient. After colonoscopy, abdominal Kampo diagnosis was determined in each patient based on medical records. Results : About half of telangiectasis-negative patients did not have the symptom of "resistance or tenderness on pressure of the para-umbilical region", whereas all telangiectasis-positive patients had this symptom $(\mathrm{p}=0.017)$. Colon polyps were observed more frequently in telangiectasis-positive patients than in telangiectasis-negative patients $(p=0.029)$. Conclusions : Livid telangiectasis on the colon mucosa may be related to "resistance or tenderness on pressure of the para-umbilical region" and Oketsu syndrome. Understanding of telangiectasis of the colon mucosa and Oketsu syndrome may contribute to the care of people's health. (173 words) (Kitakanto Med J $2003 ; 53: 165 \sim 167$ )
\end{abstract}

Key words : telangiectasis, colon, Kampo, Oketsu

\section{Introduction}

In Kampo medicine, the term Oketsu refers to a state of insufficient blood circulation and blood stasis. ${ }^{1}$ Symptoms of the Oketsu state are restlessness, insomnia, nervousness, flush, myalgia, lumbago, skin with areas of accumulated pigment (especially on the face), dark-rimmed eyes, crimson conjunctiva, spasms of peripheral blood vessels, resistance or tenderness on pressure of the para-umbilical region and the lower abdomen, dysmenorrhea, and hemorrhoids. We previously proposed a clinical diagnostic scale for the Oketsu state. ${ }^{2}$ This scale includes "Sairaku" (livid telangiectasis on the skin) as one of 17 component elements that were analyzed by stepwise regression. In Kampo medicine, livid telangiectasis of the deep lingual vein is also considered to be a symptom of Oketsu syndrome.
Japanese Kampo physicians usually place a high value on the abdominal Kampo diagnosis. " "Resistance or tenderness on pressure of the para-umbilical region" and "resistance or tenderness on pressure of the lower abdomen" are two of the Oketsu symptoms. The essential mechanism of resistance or tenderness on pressure of the para-umbilical region and the lower abdomen has not been clarified, but these symptoms might be related to blood stagnation in the abdominal space. In daily practice, we have sometimes encountered patients with both Oketsu syndrome and livid telangiectasis on the colon mucosa detected by colonoscopy (Fig. 1a, b). The object of this study was to examine the relationship between livid telangiectasis on the colon mucosa and the abdominal Kampo diagnosis.

1 Department of Integrated Japanese Oriental Medicine, Faculty of Medicine, Gunma University School of Medicine, Maebashi, Japan

2 Department of General Medicine, Gunma University School of Medicine Hospital, Maebashi, Japan

3 Department of Japanese Oriental Medicine, Faculty of Medicine, Toyama Medical and Pharmaceutical University, Toyama, Japan Received : February 12, 2003

Address: NAOKI MANTANI Department of Integrated Japanese Oriental Medicine, Faculty of Medicine, Gunma University School of Medicine, 3-39-22 Showa-machi, Maebashi, Gunma 371-8511 Japan. 


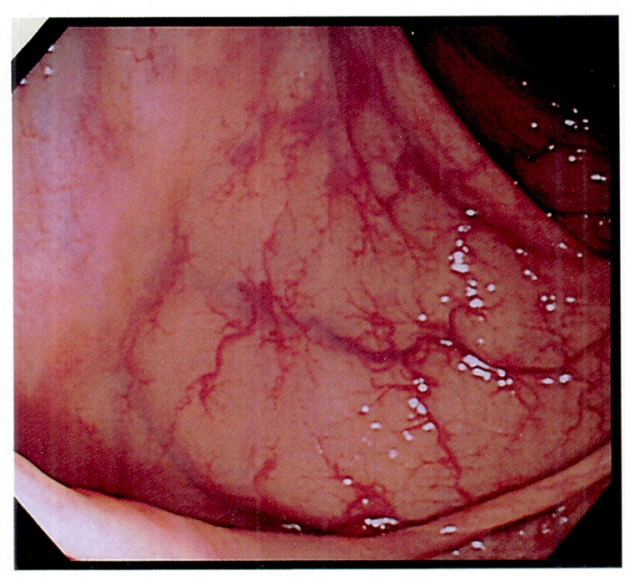

Fig. 1

\section{Patients and Methods}

We enrolled consecutive 30 patients with various diseases who underwent total colonoscopy in our department (Table 1). Patients with melanosis coli were excluded. Clinical characteristics including age, sex, colon adenoma, hemorrhoids and livid telangiectasis on the colon mucosa were determined in each patient. All endoscopic procedures were performed by one endoscopist (N.M.) with twelve years' experience who had earned a endoscopic certificate from Japan Gastroenterological Endoscopy Society. The endoscopist was blinded to information about the abdominal Kampo diagnosis made by the subject's physician. After colonoscopy, abdominal Kampo diagnoses including "abdominal tension", "resistance or tenderness on pressure" of the epigastric region, the subcostal region, the para-umbilical region, and the lower abdomen, "hypertonic rectus abdominal muscle", "sounds of fluctuating liquid", "palpitation of the abdominal aorta in the supra-umbilical region", and "reduced abdominal tension of the lower abdomen" were determined in each patient using medical records. The relationship between livid telangiectasis on the colon mucosa and abdominal Kampo diagnosis or

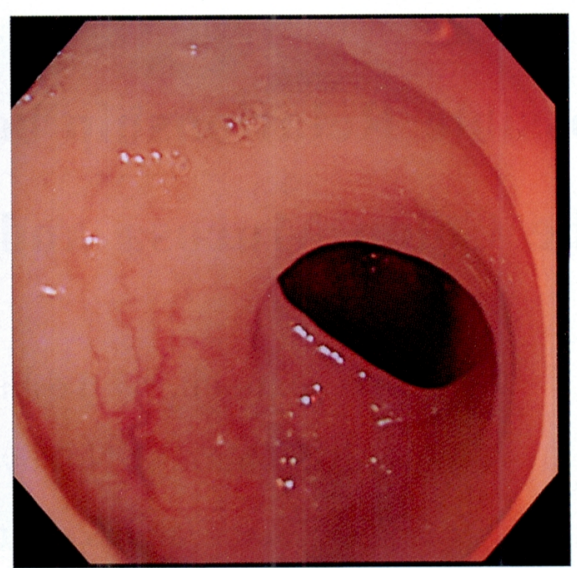

Fig. 2

clinical characteristics was analyzed. In statistical analysis, categorical variables were compared by Mann-Whitney's U test or Fisher's exact test, and continuous variables were compared by Student's ttest. A $p$-value less than 0.05 was considered statistically significant.

\section{Results}

About half of telangiectasis-negative patients did not have symptoms of "resistance or tenderness on pressure of the para-umbilical region", whereas all telangiectasis-positive patients had such symptoms $(p=0.017)$. Colon polyps were observed more frequently in the telangiectasis-positive patients than in the telangiectasis-negative patients $(\mathrm{p}=0.029)$. The telangiectasis-negative patients were more younger than the telangiectasis-positive patients $(p=0.330)$. "Resistance or tenderness on pressure of the epigastric region" was more frequently observed in the telangiectasis-positive patients than in the telangiectasis-negative patients $(p=0.215)$. None of the other clinical characteristics or abdominal Kampo diagnoses were different between the two groups (Table 1).

Table 1 Clinical characteristics and abdominal Kampo diagnosis of telangiectasis- negative and telangiectasis-positive patients

\begin{tabular}{lccc}
\hline & telangiectasis- & telangiectasis- & $p$ value \\
& negative $\mathrm{n}=16$ & positive $\mathrm{n}=14$ & $>0.9999$ \\
sex (male/female) & $7 / 9$ & $6 / 8$ & 0.330 \\
age & $49.8 \pm 18.8$ & $63.1 \pm 12.7$ & 0.570 \\
abdominal tension & $2.67 \pm 0.65$ & $2.83 \pm 1.17$ & 0.215 \\
resistance or tenderness on pressure of the epigastric region & 8 & 11 & 0.941 \\
resistance or tenderness on pressure of the subcostal region & 9 & 9 & 0.946 \\
hypertonic rectus abdominal muscle & 16 & 13 & 0.981 \\
palpitation of the abdominal aorta on the supra-umbilical & 8 & 8 & 0.017 \\
region & & 14 & 0.501 \\
resistance or tenderness on pressure of the para-umbilical & 9 & 7 & 0.093 \\
region & 5 & 12 & $>0.9999$ \\
resistance or tenderness on pressure of the lower abdomen & 8 & 1 & 0.448 \\
reduced abdominal tension of the lower abdomen & 1 & 9 & 0.029 \\
sounds of fluctuating liquid & 5 & 10 & \\
internal hemorrhoid & 4 & & \\
colon adenoma & & & \\
\hline
\end{tabular}




\section{Discussion}

Among the abdominal Kampo diagnoses studied, "resistance or tenderness on pressure of the paraumbilical region" and "colon polyps" were present at significantly different rates in the groups with and without livid telangiectasis on the colon mucosa. In Kampo medicine, both "resistance or tenderness on pressure of the para-umbilical region" and telangiectasis on the skin ("Sairaku" in Kampo medicine) are considered to be signs of Oketsu state. All patients without the sign of "resistance or tenderness on pressure of the para-umbilical region" were included in the livid telangiectasis-negative group. Therefore, livid telangiectasis on the colon mucosa may be related to "resistance or tenderness on pressure of the para-umbilical region" and Oketsu syndrome. It can be hypothesized that blood stagnation in the colon mucosa, reflected by livid telangiectasis, may cause the sign of "resistance or tenderness on pressure of the para-umbilical region".

It has been reported that the characteristic findings of colonoscopy in cirrhotic patients, collectively termed "portal hypertensive colopathy", are tree-like dilated vessels, coil-like fine vessels, vascular ectasialike lesions, and rectal varices. ${ }^{3}$ Those findings were diagnosed based on their characteristic shapes, whereas our findings were diagnosed based on livid color and telangiectasis. The livid telangiectasis in the present study was frequently accompanied by tree-like dilated vessels and coil-like fine vessels. Therefore, livid telangiectasis and "portal hypertensive colopathy" may have something in common. It is known that chronic hepatitis and liver cirrhosis are frequently accompanied by the Oketsu state. ${ }^{4}$

The telangiectasis-positive patients were older than the telangiectasis-negative patients in the present study. People with Oketsu syndrome frequently have darkrimmed eyes, areas of dark pigmentation of the facial skin, rough skin, livid lips, livid gingiva, livid tongue, and subcutaneous hemorrhage. These symptoms are more frequently observed in relatively older people. Thus, Oketsu syndrome may be more frequently observed in older people, and this may be related to the tendency for the telangiectasis on the colon mucosa to have appeared in relatively older patients in the present study.

"Resistance or tenderness on pressure of the epigastric region" tended to be more frequently observed, though not significantly so, in telangiectasis-positive patients than in telangiectasis-negative patients. Since the sign of "resistance or tenderness on pressure of the epigastric region" is not considered to be a sign of Oketsu syndrome according to traditional Kampo theory, this result was not understandable for us.

Unexpectedly, colon adenoma was observed more frequently in the telangiectasis-positive patients than in the telangiectasis-negative patients. It has been reported that the NSAID sulindac caused dramatic regression of colon adenomas in patients with familial polyposis. ${ }^{5}$ The NSAIDs are anti-inflammatory due to their ability to decrease prostaglandin synthesis by inhibiting the cyclooxygenase (COX) enzymes. A significant decrease in prostaglandin $E_{2}$ and $F_{2}$ alpha levels was seen in rectal or duodenal biopsy specimens obtained from patients treated with sulindac, and this decrease was correlated with visually determined improvement in the number and size of polyps in the same patients. ${ }^{6}$ Thus, it can be hypothesized that livid telangiectasis on the colon mucosa was induced or promoted by the vaso-dilatory effect of $\mathrm{PGE}_{2}$.

Blood vessels on the mucosa of the gastrointestinal tract from the mouth to the anus, except in the stomach, are easily observed directly or endoscopically. Understanding of telangiectasis on the mucosa of the gastrointestinal tract and Oketsu syndrome may contribute to improving the care of people's health. The relationships among the livid telangiectasis of the colon mucosa, "resistance or tenderness on pressure of the para-umbilical region", Oketsu syndrome, and colon adenoma should be examined further in a larger population.

\section{References}

1. Terasawa, K.: Kampo (Japanese-Oriental) medicine, First ed. Tokyo : K.K. Standerd McIntyre, 1993.

2. Terasawa K., Shinoda H., Imadaya A., Tosa H., Bandoh M., Satoh N. The presentation of diagnostic criteria for "Oketsu" syndrome. Jpn J Orient Med 1983; 34: 1-17 (in Japanese).

3. Motoyama H, Ueki J, Seki K, Nakamura A, Waguri N, Hashidate H, Takagi K, Hatakeyama S. Portal hypertensive colopathy : a proposal of characteristic endoscopic findings. Gastroenterological Endoscopy 1998; 40:160-168 (in Japanese).

4. Arichi S. A study of chronic hepatitis. Proc. Symp. WAKAN-YAKU $1979 ; 12: 107-113$ (in Japanese).

5. Matsuhashi N, Nakajima A, Fukushima Y, et al. Effect of sulindac on sporadic colorectal adenomatous polyps. GUT 1997; 40:344349.

6. Nugent KP, Spigelman AD, Phillips RK. Tissue prostaglandin levels in familial adenomatous polyposis patients treated with sulindac. Dis Colon Rectum 1996; 39: 659-662. 